PHYSICAL REVIEW D 95, 083501 (2017)

\title{
Spectator Higgs field, large-scale gauge fields, and the nonminimal coupling to gravity
}

\author{
Massimo Giovannini* \\ Department of Physics, Theory Division, CERN, 1211 Geneva 23, Switzerland \\ and INFN, Section of Milan-Bicocca, 20126 Milan, Italy
}

(Received 25 October 2016; published 3 April 2017)

\begin{abstract}
Even if the Higgs field does not affect the evolution of the background geometry, its massive inhomogeneities induce large-scale gauge fields whose energy density depends on the slow-roll parameters, on the effective scalar mass and, last but not least, on the dimensionless coupling to the spacetime curvature. Since the non-Abelian gauge modes are screened, the non-minimal coupling to gravity predominantly affects the evolution of the hypercharge and electromagnetic fields. While in the case of minimal coupling the obtained constraints are immaterial, as soon as the coupling increases beyond one-fourth, the produced fields become overcritical. We chart the whole parameter space of this qualitatively new set of bounds. Whenever the limits on the curvature coupling are enforced, the magnetic field may still be partially relevant for large-scale magnetogenesis and exceed $10^{-20} \mathrm{G}$ for the benchmark scale of the protogalactic collapse.
\end{abstract}

DOI: 10.1103/PhysRevD.95.083501

\section{INTRODUCTION}

According to the current evidence first established by the WMAP experiment [1], the observed temperature and polarization anisotropies are consistent with the theoretical expectations if the initial conditions of the EinsteinBoltzmann hierarchy are predominantly adiabatic and Gaussian (see also [2,3]). Every deviation from this concordance paradigm leads to entropic initial data (see e.g. [4]). When the cosmic microwave background (CMB) experiments are combined with the two remaining sets of cosmological observations $[5,6]$ the parameters describing the large-scale curvature modes (e.g. spectral index, normalization amplitude, tensor to scalar ratio) are slightly (but not crucially) affected. The adiabatic lore is then compatible with a minute tensor to scalar ratio $r_{T}$ provided some kind of plateau-like potential dominates, even before the onset of the inflationary stage, against the kinetic energy of the inflaton and against the spatial curvature [2].

Even assuming that the energy density of the Higgs field with typical mass $\mathcal{O}(125) \mathrm{GeV}$ [7] is always subdominant during the conventional inflationary evolution [8], the inhomogeneities of any spectator field are amplified during inflation and contribute, ultimately, to the total curvature budget. When the inflationary curvature scale ${ }^{1} H_{e}$ is much smaller than $10^{-6} M_{P}$ [9] nearly all the curvature perturbations observed in the microwave sky might plausibly come

\footnotetext{
*massimo.giovannini@cern.ch
}

Published by the American Physical Society under the terms of the Creative Commons Attribution 4.0 International license. Further distribution of this work must maintain attribution to the author(s) and the published article's title, journal citation, and DOI. from the quasi-flat spectrum of the Higgs field. While general arguments suggest that the inflationary rate could be lowered between $10^{-12} M_{P}$ and $10^{-7} M_{P}$ (see for instance [9]), this possibility does not apply to the specific case of the Higgs field. The lower bound on the expansion rate (i.e. $H_{e} \geq 10^{-12} M_{P}$ ) depends actually on the form of the postinflationary potential. If the quartic term dominates the potential after inflation, the corresponding energy density evolves in average as $a^{-4}$ [10] where $a$ denotes the scale factor of a Friedmann-Robertson-Walker metric. ${ }^{2}$ Consequently, if the Universe reheats suddenly after the end of inflation, the Higgs field's energy density will be negligible. Similarly, the Higgs inhomogeneities will be much smaller than the observed value of large-scale curvature perturbations [11].

The conclusion of the previous paragraph is not specific to the Higgs field, but it depends on the shape of the potential and on the postinflationary dynamics of the radiation plasma. If the evolution after inflation is instead dominated by a fluid with equation of state stiffer than radiation the ratio of the Higgs field to the background can potentially increase and the induced curvature inhomogeneities might get larger. Unconventional postinflationary evolutions of this type have been discussed long ago [9] (see also [12] for the gravitational waves produced in this context). The inflationary fluctuations of the Higgs field

\footnotetext{
${ }^{1}$ The following notations for the Planck mass will be used throughout: $\bar{M}_{P}=1 / \sqrt{8 \pi G}=M_{P} / \sqrt{8 \pi}$ where $M_{P}=$ $1.22 \times 10^{19} \mathrm{GeV}$.

${ }^{2}$ In the present paper, the background metric will always be considered conformally flat and denoted by $\bar{g}_{\mu \nu}=a^{2}(\tau) \eta_{\mu \nu}$ where $\eta_{\mu \nu}$ is the Minkowski metric and $a(\tau)$ is the scale factor typically expressed as a function of the conformal time coordinate.
} 
might also modulate the reheating process [13]. However, since it is reasonable to expect that the Higgs field will decay before becoming dominant, the induced curvature inhomogeneities could be excessively non-Gaussian, as implied by general arguments related to the dynamics of the spectator fields [9]. This last class of scenarios turns out to be strongly constrained by the observational limits on nonGaussianities [1,2].

An explicit (nonminimal) coupling to the curvature may lead to a significant production of Higgs particles [14] which have been taken to be massless both during inflation and in the subsequent radiation-dominated phase (see also [15] for earlier discussions on this issue). The contribution of the Higgs mass can be safely neglected all along the inflationary phase but it becomes important during the radiation epoch. Another potential limitation involves the gauge fields. It is well established that the evolution of the inhomogeneities of the spectator fields amplifies the gauge fields [16,17]. It is then interesting to include the coupling of the Higgs field to gravity and to compute the induced large-scale magnetic fields. Qualitatively new bounds on the coupling of the Higgs field to the spacetime curvature will emerge from these considerations. In specific corners of the parameter space, the produced gauge fields will turn out to be phenomenologically relevant (for an introduction to the problem of magnetogenesis, see, for instance, [18]).

On a general ground, large-scale magnetic fields produced during inflation can affect various phenomena and, in particular, galactic magnetism. While the correlation scale of the field must be sufficiently large (probably exceeding the Mpc at the onset of the rotation of the protogalaxy), its amplitude must not jeopardize the closure bound for all the typical scales of the problem $[16,18]$. The nonminimal coupling to the spacetime curvature (parametrized by the dimensionless constant $\xi$ ) increases the produced magnetic field for comoving scales of the order of the $\mathrm{Mpc}$ but it might also saturate the closure bound at smaller distance scales. It is then a quantitative issue to chart the corners of the parameter space where sufficiently strong magnetic fields may seed either the galactic dynamo or even the compressional amplification alone [18] (see also [19] for a classic treatise on these themes). This dual analysis, to the best of our knowledge, has not been attempted before even if there are available results in the minimally coupled case [17]. We shall show that the more general approach of this paper is fully compatible with that previous results that can be accurately recovered in the limit $\xi \rightarrow 0$. Similarly, the obtained limits will be immaterial in the conformally coupled case corresponding, within the present conventions, to the value $\xi \rightarrow-1 / 6$.

In short, the logic and the main purpose of the present investigation are the following. The produced gauge fields depend, among other things, on the specific coupling of the Higgs field to the scalar curvature. While in the case of minimal coupling (i.e. $\xi=0$ ) the constraints are negligible (see also [17]), as soon as the coupling increases the produced fields may saturate and even exceed the critical density bound. The main purpose will then be to chart the parameter space of the model with the aim of constraining the Higgs field coupling to the spacetime curvature. It will also be interesting to scrutinize more closely those regions where the fields are not overcritical but can still be relevant for the problem of magnetogenesis [18]. The layout of the paper can be summarized as follows. In Sec. II, we address the classical and quantum evolution of the Higgs field inhomogeneities by including the coupling to the spacetime curvature. In Sec. III, we shall compute the production of the massive modes and of the corresponding currents. The magnetic fields and the bounds on the curvature coupling will be discussed in Sec. IV. Section V contains the concluding remarks.

\section{HIGGS INHOMOGENEITIES}

The Higgs sector of the standard model action in a fourdimensional curved background can be written as

$S=\int d^{4} x \sqrt{-g}\left[-\frac{R}{2 \ell_{P}^{2}}+\left|D_{\mu} \hat{H}\right|^{2}-V(|\hat{H}|)-\xi R|\hat{H}|^{2}\right]$,

where $\ell_{P}=1 / \bar{M}_{P}, D_{\mu}$ is the $S U_{L}(2) \otimes U_{Y}(1)$ covariant derivative and $V(|\hat{H}|)$ is the Higgs potential, the nonminimal coupling has been denoted by $\xi$, and the complex Higgs doublet $\hat{H}$ is given in terms of four real scalar fields. In curved backgrounds, the production of scalar particles, in general (and of Higgs particles in particular), can be studied with various methods and within different approximations. The simplest approach is to disregard completely the mass and the interactions with the gauge sector. The particles produced when $\xi \neq 0$ can be computed analytically and this strategy has been followed, for instance, in Ref. [14]. Whenever $\xi$ is sufficiently close to the conformal case it is even possible to tailor a perturbative expansion whose small parameter is exactly the deviation of $\xi$ from its conformal value [20-22]. In the case of minimal coupling, $\xi$ is exactly equal to zero and the conformal expansion is reasonably well defined (see for instance the second paper in [22] and discussion therein).

The non-Abelian gauge fields are screened as the Universe thermalizes and this phenomenon has been investigated for the first time in connection with chromoelectric and chromomagnetic fields at finite temperature [23]. Conversely the hypercharge fields are not screened and remain unscreened after the electroweak phase transition $[16,17]$. Since the contribution of the Abelian fields cannot be overlooked it seems rather plausible to scrutinize their amplification and their potential effects. We shall then analyze the gauge fields induced by the massive Higgs 
fluctuations in the framework of the Abelian-Higgs model. While the mass does not affect the mode functions during inflation, it becomes essential in the radiation epoch. Conversely, in the case of a spatially flat geometry (such as the one suggested by current observations) the dimensionless parameter $\xi$ drops out from the evolution equations of the mode functions during radiation. This happens because the Ricci scalar vanishes exactly on the background.

\section{A. Abelian-Higgs model during inflation}

The total action of the Abelian-Higgs model nonminimally coupled to gravity can be expressed as

$$
\begin{aligned}
S= & \int d^{4} x \sqrt{-g}\left[-\frac{R}{2 \ell_{P}^{2}}+g^{\mu \nu}\left(\mathcal{D}_{\mu} \phi\right)^{*} \mathcal{D}_{\nu} \phi-V(|\phi|)\right. \\
& \left.-\xi R \phi^{*} \phi-\frac{1}{4} Y_{\alpha \beta} Y^{\alpha \beta}\right]
\end{aligned}
$$

where $\mathcal{D}_{\mu}=\nabla_{\mu}+i q Y_{\mu}$ is the $U(1)$ covariant derivative, $\nabla_{\mu}$ is the generally covariant derivative; $-Y_{\alpha \beta} Y^{\alpha \beta} / 4$ denotes the standard kinetic term of the gauge field. To avoid potential confusions we want to stress that $\phi$ is not the inflaton field but rather the Higgs field of the AbelianHiggs model. In this paper, we shall study the evolution of the fluctuations of the Higgs field in a fixed inflationary background described in terms of its slow-roll dynamics. The evolution equations obtained from Eq. (2.2) are (see also [24])

$$
\begin{gathered}
g^{\alpha \beta} \mathcal{D}_{\alpha} \mathcal{D}_{\beta} \phi+\frac{\partial V}{\partial \phi^{*}}+\xi R \phi=0, \\
\nabla_{\mu} Y^{\mu \nu}=j^{\nu}-2 q^{2} Y^{\nu} \phi^{*} \phi
\end{gathered}
$$

where the current appearing in Eq. (2.4) is given by

$$
j^{\nu}=g^{\mu \nu} j_{\mu}, \quad j_{\mu}=i q\left[\phi^{*} \partial_{\mu} \phi-\phi \partial_{\mu} \phi^{*}\right] .
$$

Equations (2.3) and (2.4) are supplemented by the Einstein equations which are not affected by the Higgs field during and after inflation. During inflation the total energymomentum tensor is dominated by the contribution of the inflaton whose energy density greatly exceeds the one of the Higgs boson. After inflation the energy density of the radiation background will be the leading component of the energy-momentum tensor of the plasma even if different possibilities are not excluded by the present analysis (see, for instance, Ref. [9] and discussion therein). As will be clear in Sec. III, we shall work within the sudden reheating approximation where the scale factor and the extrinsic curvature are continuous across the inflationary boundary. Since the Higgs field does not dominate at any stage of the evolution of the background it is natural to posit that $\xi \bar{R} \phi^{*} \phi \ll R \bar{M}_{P}^{2}$ where $\bar{R}$ denotes the background Ricci scalar. The previous requirement guarantees that the effects of the amplified inhomogeneities on the total curvature $R$ (i.e. background plus fluctuations) can also be ignored. In this approach, the energy density of the Higgs (or of the gauge fields) must never exceed the critical energy density.

We consider the situation where the background gauge fields are vanishing, i.e. $\bar{Y}_{\alpha}=\bar{Y}_{\alpha \beta}=0$, but their fluctuations can be dynamically generated thanks to the currents of the massive Higgs inhomogeneities [17]. To lowest order, the evolution equation of $\phi$ is only affected by $\xi$ and can be written, in general terms, as

$$
\bar{g}^{\alpha \beta} \bar{\nabla}_{\alpha} \bar{\nabla}_{\beta} \phi+m^{2} \phi+\xi \bar{R} \phi=0,
$$

where $\bar{\nabla}_{\alpha} \bar{\nabla}_{\beta}=\partial_{\alpha} \partial_{\beta}-\bar{\Gamma}_{\alpha \beta}^{\mu} \partial_{\mu}$ and $\bar{\Gamma}_{\alpha \beta}^{\mu}$ denotes the background Christoffel symbols. For the sake of accuracy, we mention that, within the present conventions, the Riemann tensor is given by $R_{\mu \alpha \nu}^{\beta}=\partial_{\alpha} \Gamma_{\mu \nu}^{\beta}+\cdots$, and the Ricci tensor is defined from the contraction of the first and third indices of the Riemann tensor, i.e. $R_{\mu \nu}=R_{\mu \alpha \nu}^{\alpha}$. The Ricci scalar of the background [in the case of a conformally flat metric $\bar{g}_{\mu \nu}=a^{2} \eta_{\mu \nu}$ with signature $\left.(+,-,-,-)\right]$ is then given by $\bar{R}=-6 a^{\prime \prime} / a^{3}$. With these specifications, Eq. (2.6) becomes

$\Phi^{\prime \prime}-\nabla^{2} \Phi-\frac{a^{\prime \prime}}{a} \Phi+m^{2} a^{2} \Phi-6 \xi \frac{a^{\prime \prime}}{a} \Phi=0, \quad \Phi=a \phi$,

and analogously for the complex conjugate field. In Eqs. (2.6) and (2.7), $m$ denotes the effective mass term and does not only necessarily coincide with the mass term of the potential, (i.e. $m_{0}$ ) but it can also include the contribution of the quartic term. Denoting with $v$ the vacuum expectation value of $|\phi|$ during inflation, whenever $v \gg \mathcal{O}(250) \mathrm{GeV}, m_{0}$ can be negligible if compared with the contribution of quartic self-interaction which is of the order of $\lambda v^{2}$.

We would like to stress that the background metric will be taken to be conformally flat. There are various reasons for this choice: first, any spatial curvature will be exponentially suppressed during inflation and, second, the concordance scenario stipulates that today the contribution of the spatial curvature to the energy budget of the Universe is negligible, meaning that we are, to a good approximation, rather close to the critical density. During inflation, however, the metric fluctuations are also amplified and the interplay between the metric fluctuations and the Higgs inhomogeneities is negligible. After inflation, the Higgs inhomogeneities might affect the large-scale curvature inhomogeneities as it happens in the case of spectator fields of different nature. This, however, does not happen. As explained in the introduction, the energy density of the 
zero mode of the Higgs field evolves like $a^{-4}$. Thus, during radiation the amplified Higgs inhomogeneities will remain small and will leave unaffected the temperature and polarization anisotropies of the cosmic microwave background [11].

What matters is the ratio between $m$ and the expansion rate both during inflation and in the subsequent radiationdominated phase. The effective mass will then enter the (four-dimensional) parameter space through the quantity $\zeta=m /\left(a_{e} H_{e}\right)$, where $H_{e}$ is the curvature scale at the end of inflation and $a_{e}$ is the scale factor at the corresponding epoch. Recall, in this respect, that the inflationary expansion rate and the inflaton potential in Planck units can be expressed as

$$
\begin{aligned}
\left(\frac{H_{e}}{M_{P}}\right) & =6.85 \times 10^{-6}\left(\frac{r_{T}}{0.1}\right)^{1 / 2}\left(\frac{\mathcal{A}_{\mathcal{R}}}{2.4 \times 10^{-9}}\right)^{1 / 2}, \\
\left(\frac{W}{M_{P}^{4}}\right) & =5.6 \times 10^{-12}\left(\frac{r_{T}}{0.1}\right)\left(\frac{\mathcal{A}_{\mathcal{R}}}{2.4 \times 10^{-9}}\right),
\end{aligned}
$$

where $\mathcal{A}_{\mathcal{R}}$ the amplitude of the scalar power spectrum at the conventional pivot wave number $k_{p}=0.002 \mathrm{Mpc}^{-1}$ and $W$ denotes the inflaton potential. If the consistency relations are enforced [as assumed in Eqs. (2.8) and (2.9)], the tensor to scalar ratio $r_{T}$, the tensor spectral index $n_{T}$, and the slow roll parameter $\epsilon$ obey the following chain of equalities $r_{T}=\mathcal{A}_{T} / \mathcal{A}_{\mathcal{R}}=16 \epsilon=-8 n_{T}$, where $\epsilon=-\dot{H} / H^{2}$ is the slow-roll parameter and $\mathcal{A}_{T}$ is the amplitude of the tensor power spectrum at the same pivot scale $k_{p}$ used to assign the scalar power spectrum. All in all, since the value of the quartic self-interaction $\lambda$ is $\mathcal{O}\left(10^{-2}\right)$, we can say, with a fair degree of confidence, that the condition $m / H_{e} \ll 1$ will always be verified in practice.

Let us now conclude this discussion by mentioning that, in Fourier space, Eq. (2.7) becomes

$$
\Phi_{\vec{k}}^{\prime \prime}+\left[k^{2}+m^{2} a^{2}-(1+6 \xi) \frac{a^{\prime \prime}}{a}\right] \Phi_{\vec{k}}=0 .
$$

In terms of $\Phi$, the total (rescaled) current of Eq. (2.5) can be expressed as

$$
J^{\mu}=\sqrt{-g} g^{\mu \nu} j_{\nu}=i q \eta^{\mu \nu}\left[\Phi^{*} \partial_{\nu} \Phi-\Phi \partial_{\nu} \Phi^{*}\right],
$$

where $\eta_{\mu \nu}$ denotes the Minkowski metric. In Eqs. (2.7) and (2.10), the cases of conformal and minimal couplings correspond, respectively, to $\xi \rightarrow-1 / 6$ and $\xi \rightarrow 0$. Note that the conformal coupling corresponds to $\xi \rightarrow+1 / 6$ in Refs. [21,22] where the Ricci scalar of the background has an opposite sign due to the different conventions defining the Riemann tensor. See also, in this respect, the discussion after Eq. (2.7).

\section{B. Action for the Higgs inhomogeneities on a fixed background}

From now on, the attention will be focused on the case of a conformally flat background geometry (i.e. $\bar{g}_{\mu \nu}(\tau)=$ $\left.a^{2}(\tau) \eta_{\mu \nu}\right)$. The action for the rescaled field $\Phi$ can be obtained from Eq. (2.2) and the explicit result is

$$
\begin{aligned}
S= & \int d^{3} x \int d \tau\left[\Phi^{* \prime} \Phi^{\prime}+\mathcal{H}^{2} \Phi^{*} \Phi-\mathcal{H}\left(\Phi^{*} \Phi^{\prime}+\Phi \Phi^{* \prime}\right)\right. \\
& \left.-m^{2} a^{2} \Phi^{*} \Phi+6 \xi\left(\mathcal{H}^{2}+\mathcal{H}^{\prime}\right) \Phi^{*} \Phi-\partial_{i} \Phi^{*} \partial^{i} \Phi\right],
\end{aligned}
$$

where $\mathcal{H}=a^{\prime} / a$ and the prime denotes a derivation with respect to $\tau$. For the action explicitly depends on time, noncovariant total derivatives can be added or subtracted without affecting the evolution equations of $\Phi$. The different results obtained in this way will lead to slightly different Hamiltonians all related by (time-dependent) canonical transformations. Indeed, recalling the obvious relation $\left(\mathcal{H} \Phi^{*} \Phi\right)^{\prime}=\mathcal{H}^{\prime} \Phi^{*} \Phi+\mathcal{H}\left(\Phi^{*} \Phi^{\prime}+\Phi \Phi^{* \prime}\right)$, each of the two terms at the right hand side of this equation can be alternatively eliminated from Eq. (2.12). Consequently two complementary forms of the Hamiltonian are given by:

$$
\begin{aligned}
H_{1}(\tau)= & \int d^{3} x\left[\Pi_{1}^{*} \Pi_{1}+\partial_{i} \Phi^{*} \partial^{i} \Phi+m^{2} a^{2} \Phi^{*} \Phi\right. \\
& +\mathcal{H}(1+6 \xi)\left(\Phi^{*} \Pi_{1}^{*}+\Phi \Pi_{1}\right) \\
& \left.+6 \xi(6 \xi+1) \mathcal{H}^{2} \Phi^{*} \Phi\right], \\
H_{2}(\tau)= & \int d^{3} x\left[\Pi_{2}^{*} \Pi_{2}+\partial_{i} \Phi^{*} \partial^{i} \Phi+m^{2} a^{2} \Phi^{*} \Phi\right. \\
& \left.-(6 \xi+1)\left(\mathcal{H}^{2}+\mathcal{H}^{\prime}\right) \Phi^{*} \Phi\right],
\end{aligned}
$$

where $\Pi_{1}$ and $\Pi_{2}$ are defined, respectively, as $\Pi_{1}=$ $\Phi^{* \prime}-\mathcal{H}(1+6 \xi) \Phi^{*}$ and as $\Pi_{2}=\Phi^{* \prime}$. The Hamilton equations derived either from Eq. (2.13) or from Eq. (2.14) are the same since the Hamiltonians are related by a canonical transformation. In the limit $\tau \rightarrow-\infty$, Eqs. (2.13) and (2.14) coincide, since, in this limit, $\Pi_{1} \sim \Pi_{2}$. If quantum-mechanical initial conditions are assigned for $\tau \rightarrow-\infty$, the state minimizing $H_{1}$ will also minimize $H_{2}$. On the contrary, when initial conditions are imposed at a finite value of the conformal time coordinate the states minimizing $H_{1}$ and $H_{2}$ might differ [25]. These differences are immaterial for the problem at hand so that we shall consistently carry on the quantization (and the evaluation of the expectation values) in terms of $\mathrm{H}_{2}$.

\section{Quantum Hamiltonian}

Promoting the classical fields to quantum operators and representing the operators in Fourier space, we can then write 


$$
\begin{aligned}
& \hat{\Phi}(\vec{x}, \tau)=\int \frac{d^{3} k}{(2 \pi)^{3 / 2}} \hat{\Phi}_{\vec{k}}(\tau) e^{-i \vec{k} \cdot \vec{x}}, \\
& \hat{\Pi}(\vec{x}, \tau)=\int \frac{d^{3} k}{(2 \pi)^{3 / 2}} \hat{\Pi}_{\vec{k}}(\tau) e^{-i \vec{k} \cdot \vec{x}},
\end{aligned}
$$

so that, from Eq. (2.14), the Hamiltonian relevant to our problem becomes

$$
\begin{aligned}
\hat{H}(\tau)= & \int d^{3} k\left[\hat{\Pi}_{\vec{k}}^{\dagger} \hat{\Pi}_{\vec{k}}+\omega_{k}^{2} \hat{\Phi}_{\vec{k}}^{\dagger} \hat{\Phi}_{\vec{k}}\right. \\
& \left.-(6 \xi+1)\left(\mathcal{H}^{2}+\mathcal{H}^{\prime}\right) \hat{\Phi}_{\vec{k}}^{\dagger} \hat{\Phi}_{\vec{k}}\right],
\end{aligned}
$$

where $\omega_{k}^{2}(\tau)=k^{2}+m^{2} a^{2}(\tau)$. For each mode of the field, Eq. (2.16) provides the quantum description of the process of parametric amplification originally analyzed in the context of quantum optics [26]. The evolution equations in the Heisenberg description can be easily obtained from Eq. (2.16):

$$
\begin{aligned}
& \partial_{\tau} \hat{\Phi}_{\vec{k}}=\hat{\Pi}_{\vec{k}}^{\dagger}, \\
& \partial_{\tau} \hat{\Pi}_{\vec{k}}^{\dagger}=-\omega_{k}^{2} \hat{\Phi}_{\vec{k}}+(1+6 \xi) \frac{a^{\prime \prime}}{a} \hat{\Phi}_{\vec{k}},
\end{aligned}
$$

and, similarly, for the Hermitian conjugate operators. The field operators $\hat{\Phi}_{\vec{k}}$ and $\hat{\Pi}_{\vec{k}}$ can be expressed in terms of the creation and annihilation operators $\hat{a}_{\vec{k}}(\tau)$ and $\hat{b}_{\vec{k}}(\tau)$, namely

$$
\begin{aligned}
& \hat{\Phi}_{\vec{k}}=\frac{1}{\sqrt{2 \omega_{k}}}\left(\hat{a}_{\vec{k}}+\hat{b}_{-\vec{k}}^{\dagger}\right), \\
& \hat{\Pi}_{\vec{k}}=-i \sqrt{\frac{\omega_{k}}{2}}\left(\hat{b}_{\vec{k}}-\hat{a}_{-\vec{k}}^{\dagger}\right) .
\end{aligned}
$$

Where $\hat{a}_{\vec{k}}$ and $\hat{b}_{\vec{p}}$ and separately obey the standard commutation relations, namely, $\left[\hat{a}_{\vec{k}}, \hat{a}_{\vec{p}}^{\dagger}\right]=\delta^{(3)}(\vec{k}-\vec{p})$ and $\left[\hat{b}_{\vec{k}}, \hat{b}_{\vec{p}}^{\dagger}\right]=\delta^{(3)}(\vec{k}-\vec{p})$. The Hermitian conjugate relations can be directly derived from Eq. (2.18).

The quantum Hamiltonian of Eq. (2.16) could now be expressed in terms of the creation and annihilation operators $\hat{a}_{\vec{k}}$ and $\hat{b}_{\vec{k}}$; the result is not diagonal but it can be brought to diagonal form by means of the following canonical transformation:

$$
\begin{aligned}
& \hat{a}_{\vec{k}}(\tau)=u_{k}(\tau) \hat{a}_{\vec{k}}\left(\tau_{i}\right)-v_{k}(\tau) \hat{b}_{-\vec{k}}^{\dagger}\left(\tau_{i}\right), \\
& \hat{b}_{-\vec{k}}(\tau)=u_{k}(\tau) \hat{b}_{-\vec{k}}\left(\tau_{i}\right)-v_{k}(\tau) \hat{a}_{\vec{k}}^{\dagger}\left(\tau_{i}\right),
\end{aligned}
$$

where $\left|u_{k}(\tau)\right|^{2}-\left|v_{k}(\tau)\right|^{2}=1$. The same canonical transformation is used to derive the ground state wave function of an interacting Bose gas at zero temperature [27]. In terms of the operators $\hat{a}_{\vec{k}}\left(\tau_{i}\right)$ and $\hat{b}_{\vec{k}}\left(\tau_{i}\right)$, the Hamiltonian is diagonal.

When the modes of the field are inside the Hubble radius at $\tau_{i}$ (i.e. $k \tau_{i} \gg 1$ ) the vacuum corresponds to the state $\left|s_{i}\right\rangle=\left|0_{a} 0_{b}\right\rangle$ which is annihilated both by $\hat{a}_{\vec{k}}\left(\tau_{i}\right)$ and by $\hat{b}_{\vec{k}}\left(\tau_{i}\right)$. From Eqs. (2.19) and (2.20), the average multiplicity of produced Higgs excitations per Fourier mode at a generic time $|\tau| \gg \tau_{i}$ is given by

$$
\begin{aligned}
\bar{n}_{k}(\tau) & =\left\langle s_{i}\left|\hat{a}_{\vec{k}}^{\dagger}(\tau) \hat{a}_{\vec{k}}(\tau)+\hat{b}_{\vec{k}}^{\dagger}(\tau) \hat{b}_{\vec{k}}(\tau)\right| s_{i}\right\rangle \\
& =2\left|v_{k}(\tau)\right|^{2} .
\end{aligned}
$$

Equation (2.21) accounts of the number of produced excitations; the particle content of the initial state will be disregarded since the average multiplicity of the produced quanta is typically very large the contribution of the initial state is immaterial for the present ends; it may be relevant to gauge the backreaction of the initial state as suggested in [25].

The evolution of $u_{k}(\tau)$ and $v_{k}(\tau)$ is related to the mode functions for the canonical fields and for the canonical momenta, i.e. $u_{k}-v_{k}^{*}=\sqrt{2 \omega_{k}} F_{k}$ and $u_{k}+v_{k}^{*}=i G_{k} \sqrt{2 / \omega_{k}}$ where $F_{k}$ and $G_{k}$ obey:

$$
\begin{aligned}
F_{k}^{\prime} & =G_{k}, \\
G_{k}^{\prime} & =-\omega_{k}^{2} F_{k}+(1+6 \xi)\left(\mathcal{H}^{2}+\mathcal{H}^{\prime}\right) F_{k} .
\end{aligned}
$$

Denoting with $\rho_{\Phi}$ the energy density of the produced inhomogeneities we also have $d \rho_{\Phi}=\omega_{k} \bar{n}_{k} d^{3} k /(2 \pi)^{3}$ which is valid in the limit $\bar{n}_{k} \gg 1$. When the background geometry expands from the inflationary stage to the radiation epoch the effective Higgs mass gives a negligible contribution during inflation. In the radiation phase, the contribution of $\xi$ can be totally disregarded (since the Ricci scalar vanishes on the background) but the mass contribution increases as $m^{2} a^{2}$ and eventually dominates the evolution of the mode functions.

\section{PRODUCTION OF MASSIVE MODES AND THEIR CURRENT}

If he slow-roll parameters are constant during the quaside Sitter phase the connection between the conformal time coordinate and the Hubble rate is simpler, namely $\mathcal{H}=$ $a H=-1 /[(1-\epsilon) \tau]$. The inflationary scale factor can then be expressed as $a_{i}(\tau)=\left(-\tau / \tau_{1}\right)^{-\beta}$ for $\tau<-\tau_{1}$, where $-\tau_{1}$ marks the end of the inflationary phase and $\beta=1$ in the case of a de Sitter phase. The scale factor of the radiation epoch hold for $\tau \geq-\tau_{1}$ and it is given by $a_{r}(\tau)=$ $\left[\beta \tau+(\beta+1) \tau_{1}\right] / \tau_{1}$. The scale factors and their first derivatives with respect to $\tau$ are continuously matched across the transition, [i.e. $a_{r}\left(-\tau_{1}\right)=a_{i}\left(-\tau_{1}\right)$ and $a_{r}^{\prime}\left(-\tau_{1}\right)=a_{i}^{\prime}\left(-\tau_{1}\right)$ ] so that the extrinsic curvature is also continuous [i.e. $\left.\mathcal{H}_{r}\left(-\tau_{1}\right)=\mathcal{H}_{i}\left(-\tau_{1}\right)\right]$. 
Instead of constructing a continuous background by direct matching, it is possible to find an interpolating background joining the two regions. This was the strategy in various applications of quantum field theory in curved backgrounds [21] and the same strategy has been used recently in a similar context [14]. In the present case, the interpolating background could be, for instance

$$
a(\tau)=a_{1}\left(\tau+\sqrt{\tau^{2}+\tau_{1}^{2}}\right) .
$$

For $\tau \ll-\tau_{1}$ the scale factor goes as $a(\tau) \simeq\left(-\tau_{1} / \tau\right)$ (quasi-de Sitter expansion); conversely, if $\tau \gg \tau_{1}, a(\tau) \simeq$ $\left(\tau / \tau_{1}\right)$ (radiation-dominated evolution). It is clear from the analytic form of Eq. (3.1) that for large negative times (i.e. $\left.\tau \ll-\tau_{1}\right) a(\tau) \simeq\left(-\tau_{1} / \tau\right)$. Conversely for $\tau \gg \tau_{1}$ we will have that $a(\tau) \simeq\left(\tau / \tau_{1}\right)$. This means that this background has the correct asymptotic behavior and it is compatible with a time dependent equation of state. Now this strategy is clearly equivalent (but probably less general) than the one adopted here where the inflationary phase is genuinely described in terms of the slow-roll parameters. The inhomogeneities of the Higgs field will clearly be amplified also in the case of Eq. (3.1) since what matters is that the expansion rate changes between two asymptotic regions of the evolution of the background.

\section{A. Nonminimal coupling in the mode functions}

Using the explicit form of the scale factor during inflation given in the previous paragraph, the solution of Eq. (2.22) implies that $F_{k}(\tau)$ and $G_{k}(\tau)$ for $\tau<-\tau_{1}$ are given by:

$$
\begin{aligned}
F_{k}(\tau)= & f_{k}(\tau)=\frac{\mathcal{N}}{\sqrt{2 k}} \sqrt{-k \tau} H_{\alpha}^{(1)}(-k \tau), \quad \tau<-\tau_{1} \\
G_{k}(\tau)= & g_{k}(\tau) \\
= & \mathcal{N} \sqrt{\frac{k}{2}} \sqrt{-k \tau}\left[H_{\alpha-1}^{(1)}(-k \tau)+\frac{(1-2 \alpha)}{2(-k \tau)} H_{\alpha}^{(1)}(-k \tau)\right], \\
& \tau<-\tau_{1},
\end{aligned}
$$

where $H_{\alpha}^{(1)}(-k \tau)$ is the Hankel function of first kind [28] with index $\alpha$ and argument $-k \tau$. In explicit terms, $\alpha$ and $\mathcal{N}$ are defined as

$$
\begin{aligned}
\alpha & =\frac{1}{2} \sqrt{1+\frac{4(2-\epsilon)(1+6 \xi)}{(1-\epsilon)^{2}}-\frac{\zeta^{2}}{(1-\epsilon)^{2}}}, \\
\mathcal{N} & =\sqrt{\frac{\pi}{2}} e^{i \frac{\pi}{4}(1+2 \alpha)},
\end{aligned}
$$

where $\zeta=m /\left(a_{e} H_{e}\right) \ll 1$ is the (constant) ratio between the mass and the inflationary expansion rate while $\epsilon$ is the standard slow-roll parameter already introduced after Eq. (2.9). To lowest order in $\epsilon$, the second relation in Eq. (3.4) implies:

$$
\alpha=\frac{3}{2} \sqrt{1+\frac{16}{3} \xi-\frac{\zeta^{2}}{9}}+\frac{\left(6-\zeta^{2}+36 \xi\right) \epsilon}{2 \sqrt{9-\zeta^{2}+48 \xi}}+\mathcal{O}\left(\epsilon^{2}\right),
$$

with the proviso that the condition $\zeta \ll 1$ is always verified in practice. For the sake of simplicity we shall require $\xi>-3 / 16$ so that the expansion (3.5) will always be well defined when $\zeta$ is negligible during inflation.

\section{B. Massive and relativistic modes}

When all the modes are ultrarelativistic (i.e. $k \gg m a$ ) the solutions of Eq. (2.22) in the radiation epoch are simply plane waves. Moreover, since $a^{\prime \prime}=0$ the mode functions are not affected by the presence of $\xi$. Over large-scales the contribution of the massive modes is comparatively more relevant. The same separation of scales occurs in the minimally coupled limit where $\xi \rightarrow 0$ [17]. The mode functions for $\tau \geq-\tau_{1}$ can be expressed as

$$
\begin{array}{ll}
F_{k}(\tau)=\mu_{k} \tilde{f}_{k}(\tau)+\nu_{k} \tilde{f}_{k}^{*}(\tau), & \tau \geq-\tau_{1}, \\
G_{k}(\tau)=\mu_{k} \tilde{g}_{k}(\tau)+\nu_{k} \tilde{g}_{k}^{*}(\tau), & \tau \geq-\tau_{1},
\end{array}
$$

where $\tilde{f}_{k}(\tau)$ and $\tilde{g}_{k}(\tau)$ are solutions of Eq. (2.22) in the radiation-dominated epoch when the scale factor is given by $a_{r}(\tau)=\left[\beta \tau+(\beta+1) \tau_{1}\right] / \tau_{1}$; the explicit expressions of $\tilde{f}_{k}(\tau)$ and $\tilde{g}_{k}(\tau)$ are:

$$
\begin{aligned}
& \tilde{f}_{k}(\tau)=\frac{1}{\sqrt[4]{2 \gamma}} e^{i \frac{\pi}{8}} \mathcal{D}_{\sigma}\left(e^{i \frac{\pi}{4}} z\right), \quad \sigma=-(i p+1 / 2), \\
& \tilde{g}_{k}(\tau)=\sqrt[4]{2 \gamma} e^{3 i \frac{\pi}{8}}\left[\frac{z}{2} e^{i \frac{\pi}{4}} \mathcal{D}_{\sigma}\left(e^{i \frac{\pi}{4}} z\right)-\mathcal{D}_{\sigma+1}\left(e^{i \frac{\pi}{4}} z\right)\right],
\end{aligned}
$$

where $\mathcal{D}_{\sigma}(x)$ are the parabolic cylinder functions with index $\sigma$ and argument $x$ [28]. Note that $\tilde{f}_{k}^{*}(z)=$ $\mathcal{D}_{i p-1 / 2}\left(e^{3 i \pi / 4} z\right) / \sqrt[4]{2 \gamma}$; the variables $z, p$ and $\gamma$ appearing in Eq. (3.8) are defined as:

$$
z=\sqrt{2 \gamma}\left[\tau+\frac{(\beta+1)}{\beta} \tau_{1}\right], \quad p=\frac{k^{2}}{2 \gamma}, \quad \gamma=\frac{\zeta \beta}{\tau_{1}^{2}} .
$$

The phases have been selected in such a way that for $z \gg|p|$ and for $k^{2} \tau_{1} \ll m, \tilde{f}_{k}(\tau) \rightarrow \sqrt{\tau_{1} /(2 m \tau)} \exp \left[-i m \tau^{2} /\left(2 \tau_{1}\right)\right]$. In terms of these variables, the evolution equation for $\tilde{f}_{k}$ can be written, from Eq. (2.22) as $d^{2} \tilde{f}_{k} / d z^{2}+$ $\left[p+z^{2} / 4\right] \tilde{f}_{k}=0$. 


\section{Mixing coefficients}

The continuous and differentiable transition ${ }^{3}$ between the inflationary phase and the radiation-dominated epoch implies that the expressions of the mode functions given in Eqs. (3.2)-(3.3) and in Eqs. (3.4)-(3.6) must also be continuous in $\tau=-\tau_{1}$. Consequently, the continuity of $F_{k}(\tau)$ and $G_{k}(\tau)$ implies $^{4}$

$$
\begin{gathered}
f_{k}\left(-\tau_{1}\right)=\mu_{k} \tilde{f}_{k}\left(-\tau_{1}\right)+\nu_{k} \tilde{f}_{k}^{*}\left(-\tau_{1}\right), \\
g_{k}\left(-\tau_{1}\right)=\mu_{k} \tilde{g}_{k}\left(-\tau_{1}\right)+\nu_{k} \tilde{g}_{k}^{*}\left(-\tau_{1}\right) .
\end{gathered}
$$

By solving this system the expression of $\mu_{k}$ and $\nu_{k}$ are functions of two dimensionless variables $m \tau_{1}$ (coinciding with $\zeta$ since $\tau_{1} \equiv \tau_{e}$ ) and $k \tau_{1}$. For the sake of accuracy it is appropriate to expand $\mu_{k}$ and $\nu_{k}$ in the limit $\zeta \ll 1$ (for fixed $\left.k \tau_{1}\right)$. The result of this manipulation is:

$$
\begin{aligned}
\mu_{k}= & \frac{e^{i \pi(\alpha+1 / 4) / 2} \Gamma(1 / 4)}{4 \beta^{1 / 4} \zeta^{1 / 4}}\left[\left(\alpha+\frac{1}{2}\right) H_{\alpha}^{(1)}\left(k \tau_{1}\right)-k \tau_{1} H_{\alpha+1}^{(1)}\left(k \tau_{1}\right)\right] \\
& -i \frac{(1+i) e^{i \pi(\alpha+1 / 4) / 2} \Gamma(3 / 4)}{2 \sqrt{2} \beta^{3 / 4}}\left[\left(\alpha+\beta+\frac{1}{2}\right) H_{\alpha}^{(1)}\left(k \tau_{1}\right)\right. \\
& \left.-k \tau_{1} H_{\alpha+1}^{(1)}\left(k \tau_{1}\right)\right] \zeta^{1 / 4}+\mathcal{O}\left(\zeta^{5 / 4}\right), \\
\nu_{k}= & -\frac{e^{i \pi(\alpha+3 / 4) / 2} \Gamma(1 / 4)}{4 \beta^{1 / 4} \zeta^{1 / 4}}\left[\left(\alpha+\frac{1}{2}\right) H_{\alpha}^{(1)}\left(k \tau_{1}\right)\right. \\
& \left.-k \tau_{1} H_{\alpha+1}^{(1)}\left(k \tau_{1}\right)\right] \\
& +\frac{(1+i) e^{i \pi(\alpha+3 / 4) / 2} \Gamma(3 / 4)}{2 \sqrt{2} \beta^{3 / 4}}\left[\left(\alpha+\beta+\frac{1}{2}\right) H_{\alpha}^{(1)}\left(k \tau_{1}\right)\right. \\
& \left.-k \tau_{1} H_{\alpha+1}^{(1)}\left(k \tau_{1}\right)\right] \zeta^{1 / 4}+\mathcal{O}\left(\zeta^{5 / 4}\right) .
\end{aligned}
$$

Equation (3.11) can be further expanded in the limit $\left|k \tau_{1}\right| \ll 1$ by recalling the small argument limit of the corresponding Hankel functions [28]. When the modes are ultrarelativistic, the corresponding mode functions are plane waves and the mixing coefficients have a well known form. ${ }^{5}$

\footnotetext{
${ }^{3}$ The pump field contains second derivatives of the scale factor. The lack of continuity of the scale factor and of its first (conformal) time derivative would imply a singularity in $a^{\prime \prime}$. If $a$ and $a^{\prime}$ are both continuous $a^{\prime \prime}$ contains, at most, a discontinuity.

${ }^{4}$ Note that the Wronskian of the solution must be conserved in the two regimes (i.e. $F_{k} G_{k}^{*}-F_{k}^{*} G_{k}=i$ ), we have that $\left|\mu_{k}\right|^{2}-\left|\nu_{k}\right|^{2}=1$.

${ }^{5}$ The mode functions $\tilde{f}_{k}(\tau)$ are plane waves with argument $k\left[\tau+(\beta+1) \tau_{1} / \beta\right]$. The dependence on $\xi$ drops also in the relativistic branch of the spectrum during the radiation epoch. The expression of the coefficients $\mu_{k}$ and $\nu_{k}$ are modified and in the case $\alpha \rightarrow 3 / 2$ they are given by $\mu_{k}=e^{2 i k \tau_{1}}\left[1-i /\left(k \tau_{1}\right)-\right.$ $\left.1 /\left(2 k^{2} \tau_{1}^{2}\right)\right]$ and $\nu_{k}=1 /\left(2 k^{2} \tau_{1}^{2}\right)$.
}

\section{Currents and average multiplicities}

With the explicit expressions of the mode functions and of the mixing coefficients the charge and the current density fluctuations can be directly computed from Eq. (2.11). The result of this calculation can be expressed in rather simple terms:

$$
\begin{aligned}
\hat{\rho}(\vec{x}, \tau)= & \frac{i q}{(2 \pi)^{3}} \int d^{3} k \int d^{3} p \\
& \times\left[\hat{\Phi}_{-\vec{k}}^{\dagger}(\tau) \hat{\Pi}_{-\vec{p}}^{\dagger}(\tau)-\hat{\Phi}_{\vec{k}}(\tau) \hat{\Pi}_{\vec{p}}(\tau)\right], \\
\hat{J}_{i}(\vec{x}, \tau)= & \frac{q}{(2 \pi)^{3}} \int d^{3} k \int d^{3} p\left(p_{i}-k_{i}\right) \hat{\Phi}_{\vec{k}}(\tau) \hat{\Phi}_{-\vec{p}}^{\dagger}(\tau) .
\end{aligned}
$$

From Eq. (3.13) we can also easily deduce that

$$
\begin{aligned}
(\vec{\nabla} \times \vec{J})_{k}= & -\frac{i q \epsilon_{i j k}}{(2 \pi)^{3}} \int d^{3} k \int d^{3} p\left(k_{i}+p_{i}\right)\left(p_{j}-k_{j}\right) \\
& \times \hat{\Phi}_{\vec{k}}(\tau) \hat{\Phi}_{-\vec{p}}^{\dagger}(\tau) e^{-i(\vec{k}+\vec{p}) \cdot \vec{x}}
\end{aligned}
$$

Using Eqs. (3.12), (3.13), and (3.14) the corresponding correlation functions can be computed. They all involve the expectation values of four field operators. For the present purposes, we shall be particularly interested in the following correlation function ${ }^{6}$

$$
\begin{aligned}
& \left\langle(\vec{\nabla} \times \vec{J})_{(\vec{x}, \tau)} \cdot(\vec{\nabla} \times \vec{J})_{(\vec{y}, \tau)}\right\rangle \\
& =\frac{q^{2}}{(2 \pi)^{6}} \int \frac{d^{3} k}{\omega_{k}} \int \frac{d^{3} k^{\prime}}{\omega_{k^{\prime}}}\left[k^{2} k^{\prime 2}-\left(\vec{k} \cdot \vec{k}^{\prime}\right)^{2}\right] \mathcal{C}\left(k, k^{\prime}, \tau\right) e^{-i\left(\vec{k}+\vec{k}^{\prime}\right) \cdot \vec{r}}, \\
& \mathcal{C}\left(k, k^{\prime}, \tau\right)=\left|u_{k}(\tau)-v_{k}^{*}(\tau)\right|^{2}\left|u_{k^{\prime}}(\tau)-v_{k^{\prime}}^{*}(\tau)\right|^{2} .
\end{aligned}
$$

Equation (3.15) and its descendants are derived by using the explicit expressions of the field operators and by evaluating the corresponding expectation values. As remarked in Eqs. (2.19) and (2.20) the initial state $\left|s_{i}\right\rangle$ is annihilated by $\hat{a}_{\vec{k}}\left(\tau_{i}\right)$ and $\hat{b}_{\vec{k}}\left(\tau_{i}\right)$ and not simply by $\hat{a}_{\vec{k}}(\tau)$ and $\hat{b}_{\vec{k}}(\tau)$.

The problem of the regularization of these correlators has been discussed in detail in Ref. [17] and we shall use here the same computational scheme. Defining the Gaussian window function $W(\vec{x}, \tau)=\exp -\left[k_{L}^{2}|\vec{x}|^{2} / 2+\omega_{L}^{2} \tau^{2} / 2\right]$ the regularized charge and current density fluctuations will be given by:

\footnotetext{
${ }^{6} \mathrm{We}$ note that the current induced by the homogeneous mode of the Higgs vanishes; this is obvious from Eqs. (3.15) and (3.16). The corresponding magnetic field will also vanish [17].
} 


$$
\begin{aligned}
(\vec{\nabla} \times \overrightarrow{\mathcal{J}})^{2}= & \Omega_{L}^{2} k_{L}^{6} \int d^{3} x \int d^{3} y \int d \tau \\
& \times \int d \tau^{\prime}\langle(\vec{\nabla} \times \vec{J}) \cdot(\vec{\nabla} \times \vec{J})\rangle W(\vec{x}, \Delta \tau) W\left(\vec{y}, \Delta \tau^{\prime}\right), \\
\mathcal{Q}^{2}= & \Omega_{L}^{2} k_{L}^{6} \int d^{3} x \int d^{3} y \int d \tau \\
& \times \int d \tau^{\prime}\left\langle\hat{\rho}(\vec{x}, \tau) \hat{\rho}\left(\vec{y}, \tau^{\prime}\right)\right\rangle W(\vec{x}, \Delta \tau) W\left(\vec{y}, \Delta \tau^{\prime}\right),
\end{aligned}
$$

where $\Delta \tau=\left(\tau-\tau_{R}\right)$ and $\Delta \tau^{\prime}=\left(\tau^{\prime}-\tau_{R}\right)$; the smearing functions select the contribution of the correlators inside a given spacetime region with typical comoving volume $V \simeq$ $1 / k_{L}^{3}$ and over a comoving time $1 / \Omega_{L} ; V$ and $\Omega_{L}$ will be chosen in such a way that $\Omega_{L}^{-3} \simeq V \ll m^{-3}$; furthermore we shall choose $\tau_{R} \geq\left|\tau_{1}\right|$ where $\tau_{1}$ denotes the inflationary boundary.

We are now going to estimate the average multiplicity of the Higgs inhomogeneities. The average multiplicity determines the energy density of the produced Higgs quanta (denoted by $\rho_{\Phi}$ hereunder) and we have to make sure that the energy density of the Higgs quanta does not exceed the energy density of the background. If this would happen the Higgs would cease to be a spectator field; this is not the case and $\rho_{\Phi}$ is always much smaller than the background contribution. Indeed, the average multiplicity of the produced Higgs particles can be computed from Eq. (2.21) by taking into account the asymptotic form of the mode functions [see Eq. (3.8)] and the results of Eq. (3.11). The final expression for the average multiplicity and for the energy density can be expressed as ${ }^{7}$

$$
\begin{aligned}
\bar{n}_{k} & \simeq \delta\left(\frac{k}{a_{e} H_{e}}\right)^{-2 \alpha} \zeta^{-1 / 2}, \\
\frac{d \rho_{\Phi}}{d \ln k} & \simeq \delta m H_{e}^{3}\left(\frac{k}{a_{e} H_{e}}\right)^{3-2 \alpha} \zeta^{-1 / 2}\left(\frac{a_{e}}{a}\right)^{3},
\end{aligned}
$$

where $\delta=\mathcal{O}\left(10^{-2}\right)$. In the case of a quasiflat spectrum (i.e. $\alpha \simeq 3 / 2$ ) up to an irrelevant logarithmic correction, the energy density of the massive quanta scales as $\rho_{\Phi}(\tau)=\delta m H_{e}^{3}\left(a_{1} / a\right)^{3}$. Because the energy density of the radiation background redshifts as $\rho_{r}(\tau) \sim$ $H_{e}^{2} M_{P}^{2}\left(a_{1} / a\right)^{4}$ we have that $\rho_{\Phi}$ will eventually exceed $\rho_{r}$ at a given time $\tau_{2}$ where $a_{2} / a_{1} \simeq\left(H_{e} / M_{P}\right)^{2} \zeta$. For instance, in the case $m=m_{0}=\mathcal{O}(125) \mathrm{GeV}$ the corresponding expansion rate would be $H_{2} \simeq \mathcal{O}\left(10^{-32}\right) H_{e}$. This potential constraint is, however, invalidated since

\footnotetext{
${ }^{7}$ Since the end of inflation coincides with the onset of the radiation epoch, we have that, by definition, $\left|k \tau_{1}\right|=\left|k /\left(a_{e} H_{e}\right)\right|$.
}

the Higgs condensate decays ${ }^{8}$ before $\tau_{2}$. The Higgs gets effectively massive at a scale $m \simeq H_{o s}$ : this equation should be viewed as a definition of $H_{o s}$. Note that $m$ [as already mentioned after Eq. (2.7)] is the effective Higgs mass. Using the results of Ref. [8], we have that $H_{o s} \simeq 0.25 \lambda^{3 / 4} H_{e}$. For $\lambda \simeq 0.01$ this result implies that $H_{o s} \simeq 8 \times 10^{-3} H_{e}$ and that $H_{o s} / H_{d e c}=\mathcal{O}(300)$ where $H_{d e c}$ denotes the expansion rate at decay (see also [8]). Consequently, since $H_{\mathrm{dec}} \simeq 2.6 \times 10^{-5} H_{e}$ we have that $H_{2} \gg H_{\text {dec }}$, as anticipated.

The considerations developed so far hold for modes $m a>k$ during the radiation epoch. The massless modes are only relevant over short wavelengths and their average multiplicity will be given, approximately, by $\bar{n}_{k} \simeq$ $\delta\left|k /\left(a_{e} H_{e}\right)\right|^{-2 \alpha}$. Recalling that $d n=\bar{n}_{k} d^{3} k /(2 \pi)^{3}$ we have that the total concentration (for simplicity in the case $\alpha \simeq 3 / 2$ ) will be, approximately, $n=\delta H_{e}^{3}\left(a_{e} / a\right)^{3}$ while their energy density will be, as usual, $\rho_{\Phi} \simeq \delta H_{e}^{4}\left(a_{e} / a\right)^{4}$.

\section{BOUNDS ON THE NONMINIMAL COUPLING}

The evolution of the gauge fields across the radiation phase is customarily analyzed by using the standard kinetic description of the plasma [29] appropriately generalized to conformally flat backgrounds [30]. While the magnetohydrodynamical treatment is only meaningful for sufficiently low frequencies, the relaxation of the initial inhomogeneities is a high-frequency phenomenon involving scales close to the plasma frequency. The regularized charge and current density fluctuations of Eq. (3.16) serve as the initial conditions of the Einstein-Vlasov system during the postinflationary evolution. In this framework, the charge fluctuations and the corresponding electric fields are efficiently dissipated but the magnetic fields are not suppressed for wave numbers smaller than the magnetic diffusivity scale $k_{\sigma} \simeq \sqrt{\sigma_{c} \mathcal{H}}$ where $\sigma_{c}$ denotes the conductivity of the postinflationary plasma. For $k<k_{\sigma}$ the Einstein-Vlasov system implies [17] that the comoving magnetic energy density relaxes to 9

\footnotetext{
${ }^{8}$ The Standard Model Higgs could decay perturbatively into quarks, leptons and gauge fields. The decay channels into weak gauge bosons and top quarks are kinematically blocked. Since the decay rate is proportional to the square of the fermion mass, the decay into bottom quarks dominate. The nonperturbative decay is much more efficient [8] and faster than its perturbative counterpart.

${ }^{9}$ The nonscreened vector modes of the hypercharge field project on the electromagnetic fields through the cosine of the Weinberg angle [denoted by $\cos \theta_{W}$ in Eq. (4.1)]. Note that the present model of magnetic field generation has nothing to do with models where the inflaton directly couples with the kinetic term of the gauge fields [31-33]. It has been recently shown that these models can be generalized to the case where the electric and magnetic gauge couplings have different evolutions [34], as it happens in the relativistic theory of Van der Waals interactions; viable scenarios free of strong coupling problems can be constructed in this framework.
} 


$$
\rho_{B} \rightarrow \frac{T^{2}}{32 \pi^{2} \bar{\alpha}^{2} n_{c}^{2}}(\vec{\nabla} \times \overrightarrow{\mathcal{J}})^{2} \cos ^{2} \theta_{W},
$$

where, as already mentioned, the currents have been regularized according to Eq. (3.16); $n_{c}(T)$ is concentration of the charged particles with average momentum $T$ and $\bar{\alpha}$ is the gauge coupling constant [17] not to be confused with the Hankel index $\alpha$ which depends on $\epsilon, \zeta$ and $\xi$ (see Eq. (3.4) and discussion thereafter). It should be mentioned that, in the present treatment, the same information contained in the energy density enters the power spectrum of the magnetic field.

A detailed description of the sudden rise of the conductivity across the inflationary transition may depend on the dynamics of reheating, on the couplings of the inflaton and even on the protoinflationary initial conditions (see e.g. [16]). The rate of equilibration of the electromagnetic reactions is, however, large in comparison with the expansion rate after inflation (and may be even bigger than the rate of the inflaton decay). Since the obtained results depend solely on the density of the charged particles and on their typical momentum, Eq. (4.1) is expected to be qualitatively applicable also when different species are not in local thermal equilibrium but only in kinetic equilibrium at different temperatures [17]. In the simplest physical situation, the protoinflationary initial data do not contain any globally neutral plasma and the number of $e$-folds is minimal or close to minimal (see Eq. (4.8) and discussion thereafter). A simple model of the conducting transition can be found, for instance, in [16] [see also Eq. (3.1)]:

$$
\begin{array}{ll}
a(\tau) & =a_{1}\left(\tau+\sqrt{\tau^{2}+\tau_{1}}\right), \quad \sigma_{\mathrm{c}}(\tau)=\frac{T}{\bar{\alpha}} \theta(\tau), \\
\theta(\tau) & =\frac{1}{8}\left(1+\frac{\tau}{\sqrt{\tau^{2}+\tau_{1}^{2}}}\right)^{3} .
\end{array}
$$

Note that $\theta(\tau)$ is a smooth representation of the Heaviside step function and $\sigma_{\mathrm{c}}(\tau)$ is the conductivity which is of the order of $T / \bar{\alpha}$ where $T$ is the temperature at the corresponding epoch. The sudden transition is recovered in the formal limit $\left|\tau_{1}\right| \rightarrow 0$.

Even if accurate analyses of the numerical value of the ratio $\left(\sigma_{c} / T\right)$ have been discussed [35], the most relevant point is that the conductivity increases linearly with the temperature. Thus the ratio $\sigma_{c} / H_{e}$ determines $k_{\sigma}$ and can then be explicitly estimated from

$$
\frac{T}{H_{e}}=\left(\frac{135}{4 \pi^{4} N_{\text {eff }}}\right)^{1 / 4}\left(\epsilon \mathcal{A}_{\mathcal{R}}\right)^{-1 / 4}
$$

where $N_{\text {eff }}$ denotes the effective number of spin degrees of freedom (in concrete estimates we shall always assume $N_{\text {eff }}=106.75$, as it happens in the standard model). In the sudden reheating approximation, the plasma right after inflation is in thermal equilibrium at $T$, up to small perturbations of the distribution functions leading to charge and current fluctuations. As the Universe decelerates after inflation, the ratio of the conductivity to the Hubble rate increases. Before proceeding further, it is interesting to note that the spectral energy density (i.e. the energy density per logarithmic interval of wave number) coincides with the magnetic power spectrum. The Fourier transform of the magnetic field intensity obeys

$$
\begin{aligned}
B_{i}(\vec{x}, \tau) & =\frac{1}{(2 \pi)^{3 / 2}} \int d^{3} k B_{i}(\vec{k}, \tau) e^{-i \vec{k} \cdot \vec{x}}, \\
\left\langle B_{i}(\vec{k}, \tau) B_{j}(\vec{p}, \tau)\right\rangle & =\frac{2 \pi^{2}}{k^{3}} P_{i j}(\hat{k}) P_{B}(k, \tau) \delta^{(3)}(\vec{k}+\vec{p}),
\end{aligned}
$$

where $P_{B}(k, \tau)$ is the magnetic power spectrum and $P_{i j}(\hat{k})=\left(\delta_{i j}-\hat{k}_{i} \hat{k}_{j}\right)$ the traceless projector. Note that $P_{B}(k, \tau)$ has dimensions of an energy density. Let us now formally compute the averaged magnetic energy density; we will have from Eq. (4.4),

$$
\rho_{B}=\frac{1}{2 a^{4}}\left\langle B^{2}\right\rangle=\frac{1}{a^{4}} \int \frac{d k}{k} P_{B}(k, \tau),
$$

where we used Eq. (4.4). Equation (4.5) defines the spectral energy density which we ought to express in units of the energy density of radiation,

$$
\Omega_{B}=\frac{1}{\rho_{r}} \frac{d \rho_{B}}{d \ln k} \equiv \frac{P_{B}(k, \tau)}{a^{4} \rho_{r}},
$$

implying that the slope of the spectral energy density coincides with the slope of the magnetic power spectrum defined according to Eq. (4.5). Since the numerator and the denominator scale in the same way in Eq. (4.6), $\Omega_{B}$ will be approximately constant during the radiation epoch. During the matter epoch, $\Omega_{B}$ will still be constant but will not coincide anymore with the magnetic energy density in critical units. The results of Eqs. (3.11) and (3.15)-(3.16), once inserted into Eq. (4.1), determine $\Omega_{B}$ through Eq. (4.6):

$$
\begin{aligned}
\Omega_{B}\left(k_{L}, \xi, \zeta, \epsilon, \mathcal{A}_{\mathcal{R}}, \beta\right)= & \left(\epsilon \mathcal{A}_{\mathcal{R}}\right)^{7 / 2} \mathcal{S}\left(\alpha, \theta_{W}\right) \mathcal{T}\left(\alpha, \beta, \epsilon, \zeta, \mathcal{A}_{\mathcal{R}}\right) \\
& \times\left(\frac{k_{L}}{a_{e} H_{e}}\right)^{10-4 \alpha}\left(\frac{m}{M_{P}}\right)^{-3}, \\
\mathcal{T}\left(\alpha, \beta, \epsilon, \zeta, \mathcal{A}_{\mathcal{R}}\right)= & \frac{9 N_{\mathrm{eff}}^{3 / 2-\alpha}}{10 \beta^{3} \bar{\alpha}}\left[(1-2 \alpha+2 \beta)^{2} \zeta \Gamma^{2}(3 / 4)\right. \\
& \left.+4(1-2 \alpha)^{2} \beta \Gamma^{2}(5 / 4)\right]^{2}, \\
\mathcal{S}\left(\alpha, \theta_{W}\right)= & 2^{2 \alpha-1} 5^{\alpha-3 / 2} 3^{2 \alpha-4} \pi^{7 / 2-2 \alpha} \\
& \times(6-4 \sqrt{2}) \Gamma^{4}(\alpha) \cos ^{2} \theta_{W}
\end{aligned}
$$

which is valid for $k_{L} /\left(a_{e} H_{e}\right) \leq m / H_{e} \equiv \zeta$. According to Eq. (4.3) $\zeta \ll k_{\sigma} /\left(a_{e} H_{e}\right)$ and $k_{\sigma} /\left(a_{e} H_{e}\right)=\mathcal{O}(1)$. If $k_{L}$ is 

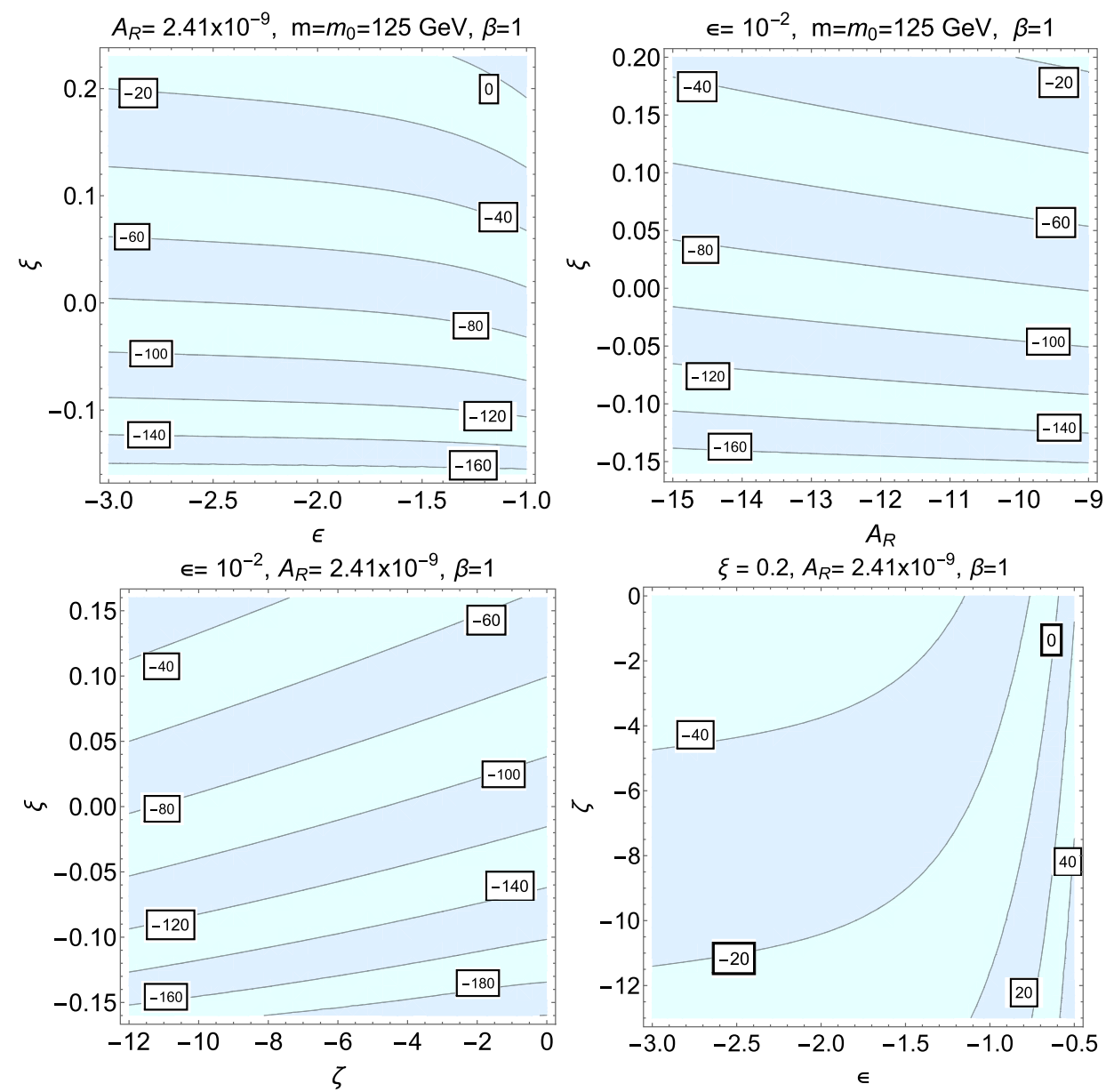

FIG. 1. The parameter space is projected on four complementary planes at the typical scale of the protogalactic collapse. The labels appearing on the curves denote the common logarithms of $\Omega_{B}$. Common logarithms are used on all the axes except for $\xi$ which is illustrated on a linear scale; $k_{L}=1 / \mathrm{Mpc}$.

evaluated at the time of the collapse of the protogalaxy we have, instead, that

$$
\begin{aligned}
\frac{k_{L}}{a_{e} H_{e}} & =\frac{k_{L}}{H_{0}} e^{-N_{\max }}, \\
e^{N_{\max }} & =\left(2 \pi \epsilon \mathcal{A}_{\mathcal{R}} \Omega_{\mathrm{R} 0}\right)^{1 / 4}\left(\frac{M_{\mathrm{P}}}{H_{0}}\right)^{1 / 2}\left(\frac{H_{r}}{H_{e}}\right)^{w-1 / 2},
\end{aligned}
$$

where $H_{0}=2.334 \times 10^{-4}\left(h_{0} / 0.7\right) \mathrm{Mpc}^{-1}$ is the present value of the Hubble rate and $\Omega_{\mathrm{R} 0}$ is the present critical fraction of radiation (in the concordance model $h_{0}^{2} \Omega_{\mathrm{R} 0}=$ $\left.4.15 \times 10^{-5}\right)$.

According to Eq. (4.7), the scale-invariant limit of the spectral energy density is obtained for $\alpha \simeq 5 / 2$. By definition this will also correspond to the scale-invariant limit of the power spectrum. This specific value of $\alpha$ corresponds to a specific value of $\xi$. More specifically, from Eq. (3.5), we will have that $\xi$ is determined, approximately, by requiring

$$
\frac{3}{2} \sqrt{1+\frac{16}{3} \xi-\frac{\zeta^{2}}{9}} \simeq \frac{5}{2} .
$$

But since $\zeta \ll 1$, we will have that the scale-invraint limit $\alpha \rightarrow 5 / 2$ is achieved when $\xi \simeq 1 / 3$. As we shall see, this value is too large and it is incompatible with the bounds discussed in Fig. 1 (see in particular the $(\epsilon, \xi)$ plane).

In Eq. (4.8), $N_{\max }$ denotes the maximal number of $e$ folds which are today accessible to our observations [36]: in practice, $N_{\max }$ is determined by fitting the redshifted inflationary event horizon inside the present Hubble radius $H_{0}^{-1}$; the term containing $w$ accounts for the possibility of a delayed reheating ending at the scale $H_{r}$ eventually much smaller than the inflationary curvature scale $H_{e}$. For illustration, we shall choose the sudden reheating approximation by setting $w=1 / 2$, but different possibilities have been considered in the literature. ${ }^{10}$ In the sudden reheating approximation, we have $N_{\max } \simeq 63.25+0.25 \ln \epsilon$ which is

\footnotetext{
${ }^{10} \mathrm{~A}$ long postinflationary phase dominated by a stiff equation of state has been examined in the context of magnetogenesis (see first paper of Ref. [36] and references therein). A delayed reheating has the effect of increasing $N_{\max }$. The largest value of $N_{\max }$ in the case of a stiff postinflationary phase can be estimated as $N_{\max }=78.3+(1 / 3) \ln \epsilon$.
} 

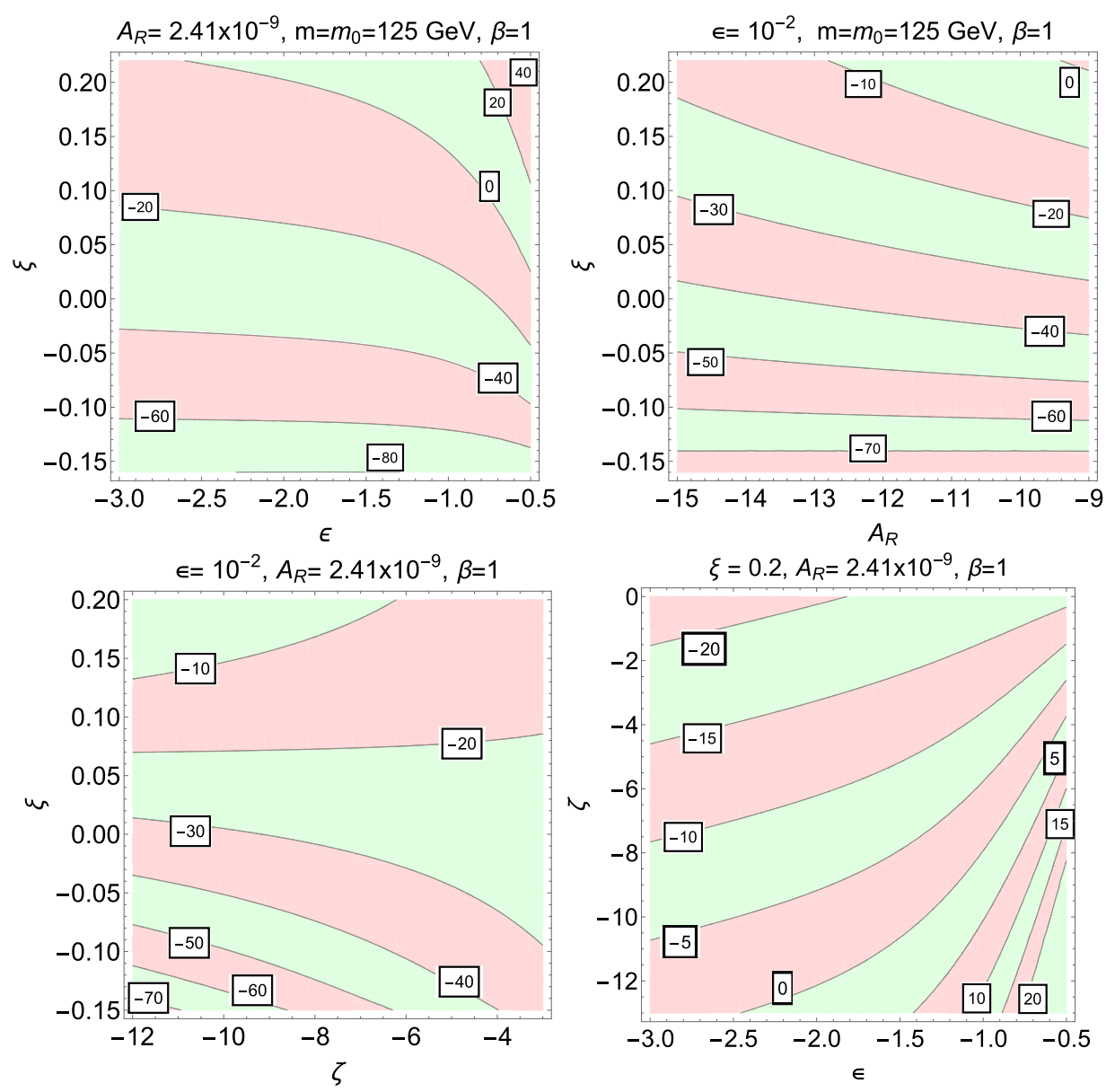

FIG. 2. We illustrate the parameter space when $k_{L}\left(a_{e} H_{e}\right)=\zeta$ which coincides with the largest wave number of the problem. The same notations adopted in Fig. 1 have been consistently employed; as in Fig. $1, k_{L}=1 / \mathrm{Mpc}$.

numerically close to the minimal number of $e$-folds needed to solve the kinematic problems of the standard cosmological model. In both Figs. 1 and 2 , we have $\beta=1$. Values $\beta \neq 1$ parametrize the deviations from the slow-roll dynamics. This situation might be interesting in its own right, but it is not central to the present discussion. ${ }^{11}$

According to Eq. (3.4), $\alpha=\alpha(\epsilon, \xi, \zeta)$ so that $\alpha$ is not an independent parameter. The parameter space is, therefore, four-dimensional and it involves $\xi, \epsilon, \zeta$, and $\mathcal{A}_{\mathcal{R}}$. The variation of $\mathcal{A}_{\mathcal{R}}$ is used to examine the regions where $H_{e}$ is artificially low in Planck units [recall, in this respect, Eqs. (2.8)-(2.9)]. In principle, there could be a further parameter, namely $\beta$. Values $\beta \neq 1$ parametrize the deviations from the slow-roll dynamics. This situation might be interesting in its own right but it is not central to the present discussion. We shall, therefore, focus on the situation $\beta=1$ where, incidentally, the term $(1-2 \alpha+2 \beta)$ is suppressed in

\footnotetext{
${ }^{11}$ The case when $\epsilon$ is not constant will have to reproduce the same quantitative features of the constant epsilon case: since the external background is fixed one can always compute the value of epsilon 50 or $60 e$-folds before the end of inflation, compute the magnetic field and discuss the various bounds.
}

Eq. (4.7) for $\xi \rightarrow 0$. In Fig. 1, the magnetic energy density in critical units is illustrated at the typical pivot scale of the protogalactic collapse (i.e. $k_{L}=\mathrm{Mpc}^{-1}$ ). Except for $\xi$, all the other quantities on the horizontal and vertical axes (i.e. $\mathcal{A}_{R}, \epsilon$ and $\zeta$ ) are reported in terms of the corresponding common logarithms. In Fig. 2, the parameter space is instead charted for $k_{L} /\left(a_{e} H_{e}\right)=\zeta$. It is useful to check, from Figs. 1 and 2, that in the minimally coupled case (i.e. $\xi \rightarrow 0$ ) the constraints are all automatically satisfied since $\Omega_{B}$ ranges from $\mathcal{O}\left(10^{-80}\right)$ to $\mathcal{O}\left(10^{-35}\right)$. The minimally coupled limit of Eq. (4.10) corresponds to the intercept $\xi=0$ in the various plots of Figs. 1 and 2. In the limit $\xi \rightarrow 0$, up to slow-roll corrections, $\alpha \rightarrow 3 / 2$ and Eq. (4.7) implies

$\Omega_{B} \simeq 1.61 \times 10^{-72}\left(\frac{k_{L}}{\mathrm{Mpc}^{-1}}\right)^{4} \cos ^{2} \theta_{W}\left(\frac{m}{\mathrm{GeV}}\right)^{-3}$,

that coincides with the previous results of Ref. [17] where $B^{2} / T^{4} \simeq 10^{-80}$ for a typical mass $m=100 \mathrm{GeV}$. At the maximal scale $k_{L} /\left(a_{e} H_{e}\right)=\zeta$ (and in the limit $\xi \rightarrow 0$ ) the magnetic energy density in critical units is instead given by 
$6.54 \cos ^{2} \theta_{W}\left(m / M_{P}\right)\left(\mathcal{A}_{\mathcal{R}} \epsilon\right)^{3 / 2}$ which is never overcritical. As previously established in the case $\xi \rightarrow 0$ there are practically no constraints. From the $(\xi, \epsilon)$ plane (plot at the top left in Fig. 1) the closure bound is violated for $\xi>\mathcal{O}(0.25)$ and $\epsilon<\mathcal{O}\left(10^{-3}\right)$. The $\left(\xi, \mathcal{A}_{\mathcal{R}}\right)$ plane illustrates, as already mentioned, the situation where the inflationary scale is artificially lowered as in [9]. The consistency relations do not necessarily hold in this case but the values of the magnetic fields are even smaller and there are no constraints on $\xi$. When charting the $\left(\xi, \mathcal{A}_{\mathcal{R}}\right)$ plane we set the effective mass to its minimal value which is the most constraining: larger values of the mass will suppress the magnetic energy density even further. A similar conclusion emerges from the analysis of the $(\xi, \zeta)$ plane.

If the magnetic energy density is approximately larger than $10^{-22} \mathrm{nG}^{2}$ at the scale of the protogalactic collapse, the observed magnetic fields could be subsequently amplified by the combined action of the gravitational collapse itself and by the galactic rotation which transforms the kinetic energy of the plasma into magnetic energy (this is the meaning of the so-called galactic dynamo [19] which has a long history [18]). The most optimistic estimates for the required initial conditions are derived by assuming that every rotation of the galaxy would increase the magnetic field of one $e$-fold. The number of galactic rotations since the collapse of the protogalaxy can be between 30 and 35, leading approximately to a purported growth of 13 orders of magnitude (see [18] and discussions therein). If the dynamo action is totally absent, the required field should be $\mathcal{O}\left(10^{-2}\right) \mathrm{nG}$ which means $\Omega_{B}=\mathcal{O}\left(10^{-10}\right)$. In this case, during the collapse of the protogalaxy, the magnetic field will increase by about 5 orders of magnitude. The shaded regions in Fig. 3 illustrate the interplay between the closure bounds and the magnetogenesis requirements. The seed field at the onset of the gravitational collapse must be, at least, as large as $10^{-15} \mathrm{nG}$ or, more realistically, larger than $10^{-11} \mathrm{nG}$. The simplest way to implement the magnetogenesis requirements is to plot the magnetic energy density for all the regions where the backreaction constraints are satisfied. The areas of the parameter space where $\log \Omega_{B}>-25$ (or, even more optimistically, $\log \Omega_{B}>-35$ ) will therefore offer viable models of magnetogenesis.

In Fig. 3, we illustrate the region where the closure bounds are satisfied and $\Omega_{B}>10^{-25}$. In these regions, the magnetogenesis requirements are met for $0.2 \leq \xi<0.24$ and $\epsilon<0.01$. The allowed regions of Fig. 3 shrink almost to a point if we enforce the closure bound and demand, at the scale of the protogalactic collapse, $\log \Omega_{B}>-14$. If we impose an even more demanding constraint (i.e. $\left.\log \Omega_{B}>-11\right)$ the allowed regions disappear completely. If $\Omega_{B}=\mathcal{O}\left(10^{-11}\right)$ the magnetic field of the galaxy could be obtained almost completely by compressional amplification [18]. Compressional amplification alone is therefore insufficient and to obtain the observed fields some sort of dynamo action is mandatory, at least in the present framework. A more detailed account of these themes is beyond the scope of this paper but can be found in more comprehensive reviews describing the features of our magnetized universe [18] (see, in particular, first and second reviews). Note, finally, that direct constraints can be obtained from CMB data (see, for instance, [37] for some recent analyses obtained from the temperature and polarization anisotropies). Indeed, as noticed over ten years ago, the temperature and the polarization anisotropies of the CMB may be magnetized since the initial conditions of the scalar modes are affected by the presence of stochastic magnetic fields [38]. This observation offers the unique opportunity of direct limits on the large-scale magnetism prior to matter-radiation equality since the large-scale magnetic fields affect directly the initial conditions of the Einstein-Boltzmann hierarchy. The current Planck explorer data have been used to set bounds on large-scale magnetic fields coming from inflation as previoulsy done with the WMAP 3-yr and 9-yr releases (see, respectively,
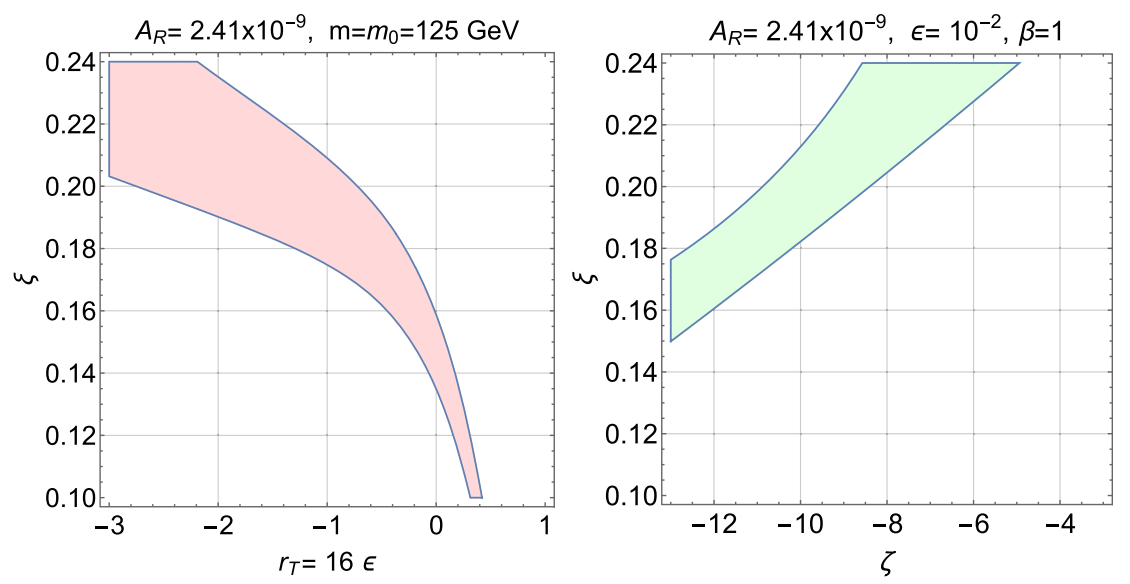

FIG. 3. We illustrate the magnetogenesis constraints in the planes $(\xi, \epsilon)$ and $(\xi, \zeta)$. The filled areas denote the allowed regions. 
third and fourth papers of Ref. [38]). The allowed regions of Fig. 3 are abundantly compatible with the CMB constraints.

\section{CONCLUDING REMARKS}

We analyzed the parametric amplification of massive Higgs excitations when the coupling to gravity is nonminimal. In the framework of the Abelian-Higgs model, this process may lead to overcritical large-scale gauge fields. As a consequence, the nonminimal coupling cannot be too large (i.e. typically $\xi \leq 0.25$ ) when $\epsilon<\mathcal{O}\left(10^{-2}\right)$.
When the coupling is either minimal (i.e. $\xi \rightarrow 0$ ) or conformal (i.e. $\xi \rightarrow-1 / 6$ ), there are practically no constraints. A global scan of the parameter space suggests that the large-scale magnetic fields produced when $\xi=\mathcal{O}(0.2)$ turn out to be partially relevant for the problem of primordial magnetogenesis.

\section{ACKNOWLEDGMENTS}

It is a pleasure to thank A. Gentil-Beccot and S. Rohr for their kind help.
[1] D. N. Spergel et al., Astrophys. J. Suppl. Ser. 148, 175 (2003); 170, 377 (2007); L. Page et al., ibid. 170, 335 (2007).

[2] B. Gold et al., Astrophys. J. Suppl. Ser. 192, 15 (2011); D. Larson et al., ibid. 192, 16 (2011); C. L. Bennett et al., ibid. 192, 17 (2011); G. Hinshaw et al., ibid. 208, 19 (2013); C. L. Bennett et al., ibid. 208, 20 (2013); P. A. R. Ade et al. (Planck Collaboration), Astron. Astrophys. 571, A22 (2014); 571, A16 (2014).

[3] C. L. Reichardt, P. A. R. Ade, J. J. Bock et al., Astrophys. J. 694, 1200 (2009); M. Zemcov et al. (QUaD Collaboration), Astrophys. J. 710, 1541 (2010).

[4] K. Enqvist, H. Kurki-Suonio, and J. Valiviita, Phys. Rev. D 62, 103003 (2000); J. Valiviita and V. Muhonen, Phys. Rev. Lett. 91, 131302 (2003); H. Kurki-Suonio, V. Muhonen, and J. Valiviita, Phys. Rev. D 71, 063005 (2005); M. Giovannini, Classical Quantum Gravity 23, 4991 (2006).

[5] W. J. Percival, B. A. Reid, D. J. Eisenstein et al., Mon. Not. R. Astron. Soc. 401, 2148 (2010); S. Roychowdhury, J. N. Chengalur, A. Begum, and I. D. Karachentsev, Mon. Not. R. Astron. Soc. 404, L60 (2010).

[6] R. Kessler, A. Becker, D. Cinabro et al., Astrophys. J. Suppl. Ser. 185, 32 (2009); M. Hicken, W. M. Wood-Vasey, S. Blondin, P. Challis, S. Jha, P. L. Kelly, A. Rest, and R. P. Kirshner, Astrophys. J. 700, 1097 (2009).

[7] G. Aad et al. (ATLAS Collaboration), Phys. Lett. B 716, 1 (2012); S. Chatrchyan et al. (CMS Collaboration), Phys. Lett. B 716, 30 (2012).

[8] K. Enqvist, T. Meriniemi, and S. Nurmi, J. Cosmol. Astropart. Phys. 10 (2013) 057; 07 (2014) 025.

[9] M. Giovannini, Phys. Rev. D 67, 123512 (2003); D. H. Lyth, Phys. Lett. B 579, 239 (2004); Phys. Rev. D 69, 083509 (2004).

[10] M. S. Turner, Phys. Rev. D 28, 1243 (1983).

[11] K. Y. Choi and Q. G. Huang, Phys. Rev. D 87, 043501 (2013).

[12] M. Giovannini, Phys. Rev. D 58, 083504 (1998); 60, 123511 (1999); Classical Quantum Gravity 26, 045004 (2009).

[13] D. H. Lyth, J. Cosmol. Astropart. Phys. 11 (2005) 006; Phys. Rev. Lett. 97, 121301 (2006).

[14] M. Herranen, T. Markkanen, S. Nurmi, and A. Rajantie, Phys. Rev. Lett. 115, 241301 (2015).
[15] T. Muta and S. D. Odintsov, Mod. Phys. Lett. A 06, 3641 (1991); S. Mukaigawa, T. Muta, and S. D. Odintsov, Int. J. Mod. Phys. A 13, 2739 (1998).

[16] M. Giovannini, Phys. Lett. B 659, 661 (2008); Phys. Rev. D 85, 101301 (2012); 86, 103009 (2012).

[17] M. Giovannini and M. E. Shaposhnikov, Phys. Rev. D 62, 103512 (2000).

[18] K. Enqvist, Int. J. Mod. Phys. D 07, 331 (1998); M. Giovannini, Int. J. Mod. Phys. D 13, 391 (2004); J. D. Barrow, R. Maartens, and C. G. Tsagas, Phys. Rep. 449, 131 (2007).

[19] E. N. Parker, Cosmical Magnetic Fields (Clarendon Press, Oxford, 1979).

[20] Ya. B. Zel'dovich and A. A. Starobinsky, Zh. Eksp. Teor. Fiz. 61, 2161 (1971) [Sov. Phys. JETP 34, 1159 (1972)]; Pis'ma Zh. Eksp. Teor. Fiz. 26, 373 (1977) [JETP Lett. 26, 252 (1977)].

[21] N. D. Birrell and P.C.W. Davies, Quantum Fields in Curved Space (Cambridge University Press, Cambridge, England, 1982).

[22] L. H. Ford, Phys. Rev. D 35, 2955 (1987); E. D. Schiappacasse and L.H. Ford, Phys. Rev. D 94, 084030 (2016).

[23] A. Linde, Phys. Lett. 96B, 289 (1980); D. J. Gross, R. D. Pisarski, and L. G. Yaffe, Rev. Mod. Phys. 53, 43 (1981).

[24] L. H. Ford, Phys. Rev. D 31, 704 (1985).

[25] M. Giovannini, Classical Quantum Gravity 20, 5455 (2003).

[26] B. L. Mollow and R. J. Glauber, Phys. Rev. 160, 1076 (1967); 160, 1097 (1967); D. Stoler, Phys. Rev. D 1, 3217 (1970); 4, 1925 (1971).

[27] A. L. Fetter and J. D. Walecka, Quantum Theory of ManyParticle Systems (McGraw-Hill, New York, 1971); A. I. Solomon, J. Math. Phys. (N.Y.) 12, 390 (1971).

[28] A. Erdelyi, W. Magnus, F. Oberhettinger, and F. R. Tricomi, Higher Trascendental Functions (Mc Graw-Hill, New York, 1953); M. Abramowitz and I. A. Stegun, Handbook of Mathematical Functions (Dover, New York, 1972).

[29] T. J. M Boyd and J. J. Sanderson, The Physics of Plasmas, (Cambridge University Press, Cambridge, England, 2003).

[30] J. Bernstein, Kinetic Theory in the Expanding Universe, (Cambridge University Press, Cambridge, England, 1988). 
[31] B. Ratra, Astrophys. J. Lett. 391, L1 (1992); M. Gasperini, M. Giovannini, and G. Veneziano, Phys. Rev. Lett. 75, 3796 (1995); M. Giovannini, Phys. Rev. D 56, 3198 (1997); M. Giovannini, Phys. Rev. D 64, 061301 (2001).

[32] K. Bamba and M. Sasaki, J. Cosmol. Astropart. Phys. 02 (2007) 030; K. Bamba, J. Cosmol. Astropart. Phys. 10 (2007) 015; M. Giovannini, Phys. Lett. B 659, 661 (2008).

[33] K. Bamba, Phys. Rev. D 75, 083516 (2007); J. Martin and J. 'i. Yokoyama, J. Cosmol. Astropart. Phys. 01 (2008) 025; M. Giovannini, Lect. Notes Phys. 737, 863 (2008); S. Kanno, J. Soda, and M.-a. Watanabe, J. Cosmol. Astropart. Phys. 12 (2009) 009; L. Campanelli, Phys. Rev. D 93, 063501 (2016); L. Campanelli and A. Marrone, Phys. Rev. D 94, 103510 (2016).

[34] M. Giovannini, Phys. Rev. D 88, 083533 (2013); 92, 043521 (2015); 92, 121301 (2015); G. Tasinato, J. Cosmol.
Astropart. Phys. 03 (2015) 040; R. Z. Ferreira and J. Ganc, J. Cosmol. Astropart. Phys. 04 (2015) 029; M. Giovannini, Phys. Rev. D 93, 043543 (2016).

[35] J. Ahonen, Phys. Rev. D 59, 023004 (1998); J. Ahonen and K. Enqvist, Phys. Lett. B 382, 40 (1996); H. Heiselberg, Phys. Rev. D 49, 4739 (1994).

[36] M. Giovannini, Phys. Rev. D 86, 103009 (2012); 87, 083004 (2013); Classical Quantum Gravity 21, 4209 (2004); A. R. Liddle and S.M. Leach, Phys. Rev. D 68, 103503 (2003).

[37] P. A. R. Ade et al. (Planck Collaboration), arXiv:1502.01594; P. A. R. Ade et al. (POLARBEAR Collaboration), Phys. Rev. D 92, 123509 (2015).

[38] M. Giovannini, Classical Quantum Gravity 23, R1 (2006); Phys. Rev. D 74, 063002 (2006); 79, 121302 (2009); Classical Quantum Gravity 30, 205017 (2013). 